\title{
Contrast Sensitivity Comparison of Alternate Exotropia in Different Amount of Deviation
}

\section{Partha Haradhan Chowdhury ${ }^{*}$ and Brinda Haren Shah ${ }^{2}$}

1M. Optom, Department of Optometry, Shree Satchandi Jankalyan Samiti Netra Prasikshan Sansthan, Pauri, Affiliated to Uttarakhand State Medical Faculty, India 2M. Optom, Department of Optometry, Gujarat University, India

\section{Research Article \\ Volume 3 Special Issue 1}

Received Date: August 02, 2018

Published Date: September 20, 2018

*Corresponding author: Partha Haradhan Chowdhury, M. Optom, Department of Optometry, Shree Satchandi Jankalyan Samiti Netra Prasikshan Sansthan, Pauri, Affiliated to Uttarakhand State Medical Faculty, Dehradun, India, Email: optometrypublish@gmail.com

\section{Abstract}

Purpose: Aim of the present study is to compare contrast sensitivity in different amounts of deviation of alternate esotropia.

Methods: A pilot, cross sectional, observational study was performed at tertiary eye care centers. Subjects with Ocular deviation between 10 to 40 prism diopters, Corrected distance Visual Acuity should be greater than 6/18 and Age should be between 10 to 40 years of age were included in the study. Contrast sensitivity was assessed with Pelli Robson Chart.

Results: 30 subjects were included in the study. Out of that, 16 subjects were in the age group of 11-20 years, 12 subjects were in the age group of 21-30 years and 2 subjects were in the age group of $31-40$ years. $60 \%$ subjects were Female and $40 \%$ subjects were Male. The mean contrast sensitivity was considered in each amount of deviation. It shows that maximum contrast is deteriorated in the ocular deviation of 31-40 prism diopters.

Conclusions: As the amount of ocular deviation increases, contrast sensitivity will be more deteriorated.

Keywords: Contrast Sensitivity; Amount of deviation; Alternate Exotropia

\section{Introduction}

Ocular deviation is a very important factor for contrast sensitivity. In cases of ocular deviation, the images of an object are falling on the parafoveal region. In case of Eso deviation, the images of an object are falling to the nasal side of the retina and in case of Exo deviation; the images of an object are falling to the temporal side of the retina. The ocular deviation is become very crucial due to anatomical consideration because cone cells vary from area to area. Cone cells are highest at the foveal region as compared to para foveal region and cone cells are fully responsible for contrast sensitivity. So, in case of ocular deviation, there are chances for deterioration of contrast sensitivity due to cone cells variation.

In cases of alternate Exoropia, the images are focused alternately at the temporal parafoveal region. As cone cell density is very low, there are strong chances of deterioration of contrast sensitivity. 


\section{Methodology}

Pilot, Cross Sectional and observational study were performed at tertiary eye care centers. Inclusion criteria includes Subjects with Ocular deviation between 10 to 40 prism diopters, Corrected distance Visual Acuity should be greater than 6/18 and Age should be between 10 to 40 years of age. Individuals with any other systemic disease(specially which can affect study), Individuals with any other Ocular Pathology, with any active ocular infection, any ocular anomalies like Corneal Scar etc ,ocular deviation if less than 10 degree and Significant amount of amblyopic patient were excluded from the study. Full refractive correction along with detailed fundus evaluation was performed in each and every patient. Visual Acuity was assessed with Log Mar Chart in different amounts of deviation of Alternate Exotropia. Data analysis was done using SPSS software version 20 .

\section{Results}

30 subjects were included in the study. Age wise distribution is shown in Figure 1. 16, 12 and 2 subjects were in the age group of 11-20 years, 12 in 21-30 years and 2 subjects were in the age group of 31-40 years. Figure 2 shows gender wise distribution of the subjects. $60 \%$ subjects were Female and $40 \%$ subjects were Male. Figure 3 shows comparison of mean Contrast Sensitivity of subjects with Alternate Exotropia with different amount of ocular deviation. In Alternate Exotropia of 1120 prism diopters, mean Contrast Sensitivity is 1.95 . In Alternate Exotropia of $21-30$ prism diopters, mean Contrast Sensitivity is 1.87.In Alternate Exotropia of 3140 prism diopters, mean Contrast Sensitivity is 1.8.This shows that as amount of ocular deviation increases, Contrast Sensitivity deteriorates [1-3].

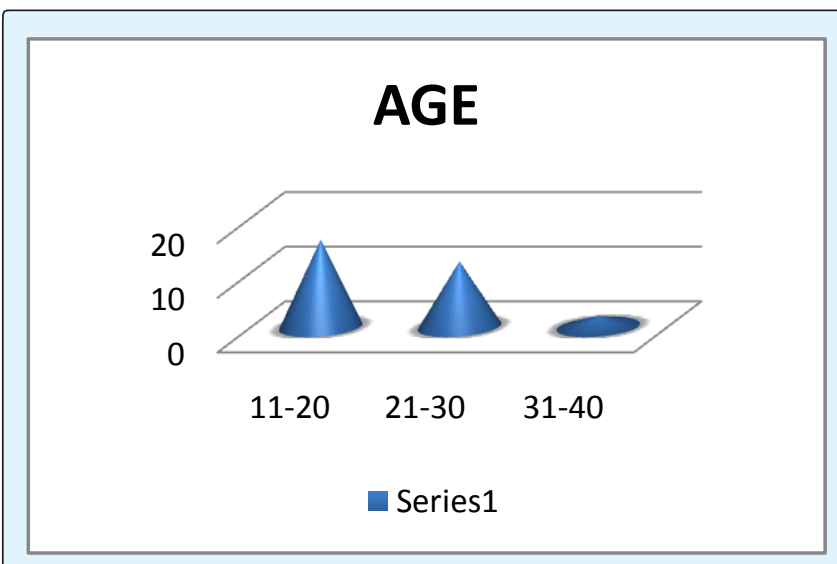

Figure 1: Shows age wise distribution of the Subject.

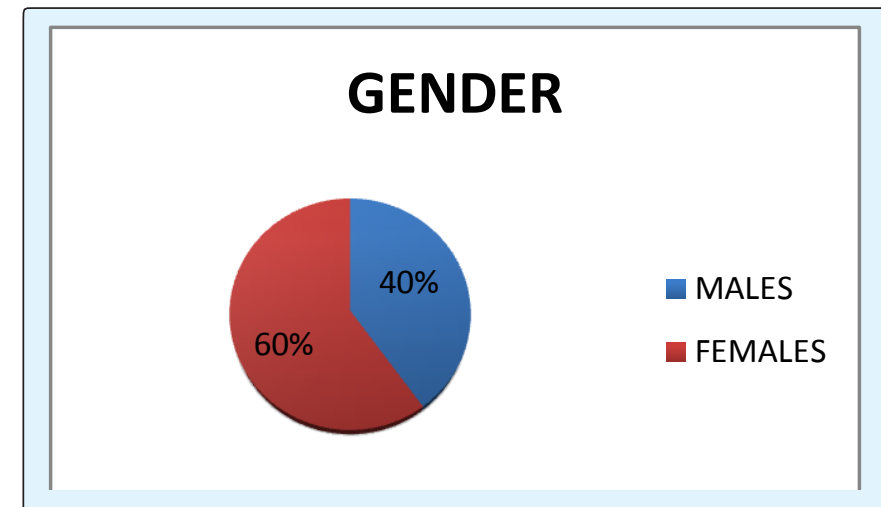

Figure 2: Shows gender wise distribution of the subjects.

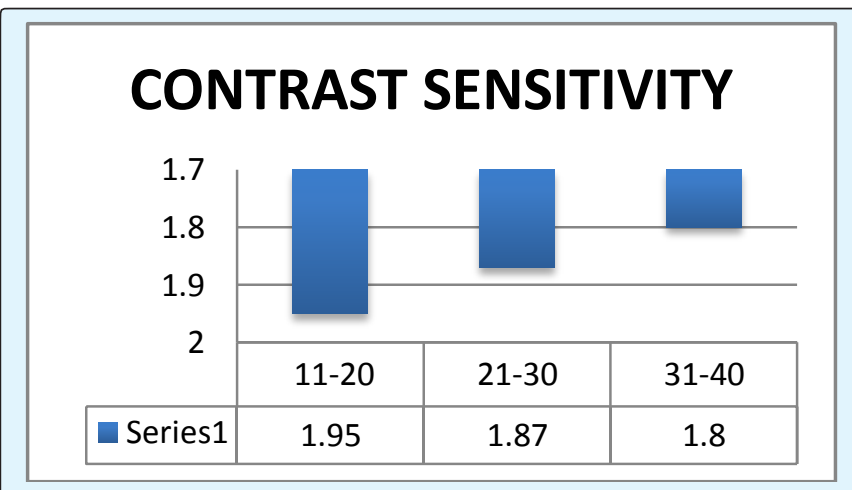

Figure 3: Shows comparison of Contrast Sensitivity for different amount of deviation for Alternate Exotropia.

\begin{tabular}{|c|c|}
\hline Contrast Sensitivity & Log Units \\
\hline $11-20$ & 1.95 \\
\hline $21-30$ & 1.87 \\
\hline $31-40$ & 1.8 \\
\hline
\end{tabular}

Table 1: Contrast Sensitivity.

\section{Discussion}

Ocular deviation is a very important factor for all ocular parameters like visual acuity, colour vision as well as contrast sensitivity. In case of Alternate Exo deviation, images are focused alternately at the temporal parafoveal region. And due to anatomical consideration and cone cells variation over the macula. Like other parameters Contrast Sensitivity is being deteriorated [4-7].

\section{Conclusions}

As the amount of ocular deviation increases, contrast sensitivity will be more deteriorated.
Partha Haradhan Chowdhury and Brinda Haren Shah. Contrast Sensitivity Comparison of Alternate Exotropia in Different Amount of Deviation. J Ophthalmol 2018, 3(S1): 000S1-010.
Copyright@ Partha Haradhan Chowdhury and Brinda Haren Shah. 


\section{Open Access Journal of Ophthalmology}

\section{Consent}

Oral/ written consent was obtained from patient as well as from tertiary eye care centres.

\section{References}

1. Kenneth $\mathrm{W}$ Wright, Peter H Spiegel, Lisa Thompson (2006) Handbook of Pediatric Strabismus and Amblyopia. 1 ${ }^{\text {st }}$ (Edn.).

2. Hui Zhu, Jia-Jia Yu, Rong-Bin Yu, Hui Ding, Jing Bai, et al. (2015) Association between Childhood Strabismus and Refractive Error in Chinese Preschool Children. Plos One 10(3): e0120720.

3. Zhale Rajavi, Sabbaghi H, Baghini AS, Yaseri M, Sheibani K, et al. (2015) Prevalence of Colour Vision Deficiency and its Correlation with Amblyopia and Refractive Errors among Primary School Children.
Journal of Ophthalmic and Vision Research 10(2): 130-138.

4. Anika K Tandon, Federico G Velez, Sherwin J Isenberg, Joseph L Demer, Stacy L Pineles (2014) Binocular Inhibition in Strabismic Patients is Associated with Diminished Quality of Life. JAAPOS 18(5): 423-426.

5. Ye XC, Pegado V, Patel MS, Wasserman WW (2014) Strabismus genetics across a spectrum of eye misalignment disorders. Clinical Gene 86(2): 103111.

6. Kocak-Altintas AG (2000) Colour vision and Colour Vision deficiency in Amblyopia. European Journal of Ophthalmology 10(1): 77-81.

7. Alan W Freeman, Nguyen VA, Jolly N (1996) Components of Colour vision Loss in Strabismus. Journal of vision research 36(5): 765-774.

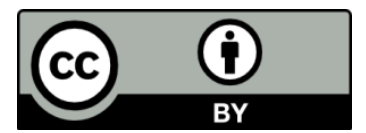

Partha Haradhan Chowdhury and Brinda Haren Shah. Contrast Sensitivity Comparison of Alternate Exotropia in Different Amount of Deviation. J Ophthalmol 2018, 3(S1): 000S1-010.
Copyright $($ Partha Haradhan Chowdhury and Brinda Haren Shah. 